\title{
Estrategia adaptativa del leño Aspidosperma quebracho blanco
}

\author{
Juana Graciela Moglia ${ }^{1}$ \\ Carlos Raúl López ${ }^{1}$
}

\begin{abstract}
RESUMEN
Se analizó la asociación entre variables anatómicas del leño y las relaciones funcionales en el xilema de Aspidosperma quebracho-blanco para estudiar si los elementos asociados con la resistencia mecánica también intervienen en la conducción. Para ello se utilizó el método de correlaciones canónicas. Se seleccionaron las variables área, diámetro y longitud de elementos de vasos como variables del conjunto dependiente para explicar la conducción y las variables diámetro, espesor de pared, longitud y porcentaje de fibras como variables del conjunto independiente asociadas a la resistencia con el supuesto de que ellas también intervienen en la conducción. Los resultados mostraron que ambos conjuntos están altamente correlacionados denotando la asociación entre los sistemas de conducción y la resistencia mecánica en el xilema. El quebracho blanco presenta un xilema muy especializado para tolerar el estrés hídrico. Los vasos solitarios proporcionan eficiencia en la conducción mientras que las traqueidas vasicéntricas y fibrotraqueidas forman un sistema subsidiario e intervienen también proporcionando seguridad en la conducción. En esta especie el xilema funciona como de una red interconectada.
\end{abstract}

PALABRAS CLAVE:

Aspidosperma, estrategia adaptativa, xilema, correlaciones canónicas, quebracho-blanco.

\begin{abstract}
In order to study if mechanical elements are involved in conduction, relationships between wood anatomical variables and functional relations were analyzed. For this purpose canonical correlation analysis was performed. Vessel area, diameter and length were selected as the dependant set to represent xylem conductivity, and fiber diameter, length and wall thickness as well as fiber percentage as the independent set, associated to mechanical elements, assuming that they are involved in conduction too. Results showed that both sets of variables were highly correlated showing the association between conduction and mechanical systems. Aspidosperma quebracho-blanco has specialized xylem to avoid water stress. Solitary vessels provide efficient water conduction, while tracheids and fiber-tracheids provide a subsidiary system responsible also of water movement. In this species xylem works as an interconnected network in which conductive and resistance systems are interrelated.
\end{abstract}

KEY WORDS:

Aspidosperma, adaptive strategy, xylem, canonical correlation, quebracho-blanco. 


\section{INTRODUCCIÓN}

La anatomía de una especie es el resultado entre dos fuerzas de selección contrapuestas: una tiende a incrementar la eficiencia conductora del agua en el xilema y la otra a proporcionar una conducción más segura (Zimmermann, 1983).

El xilema es el tejido responsable de transportar agua cuando el árbol está vivo, por lo tanto su estructura debe estar en concordancia para que este transporte sea lo más eficiente y seguro posible.

En las regiones tropicales húmedas, lo más importante es la eficiencia en el transporte ya que la disponibilidad de agua no es un factor limitante. Las especies arbóreas que la habitan tienen su tejido xilemático adaptado para conducir volúmenes grandes de agua en poco tiempo. En consecuencia, es común encontrar maderas con vasos medianos a grandes, poco numerosos y elementos de vaso largos. Los elementos de vaso anchos contribuyen a una conducción más eficiente, aunque más vulnerable, que los vasos pequeños (Carlquist, 1975; Baas, 1976; Zimmermann, 1982; Tyree et al., 1994). Los elementos de vasos largos están correlacionados con hábitats más mésicos (Carlquist, 1975).

El xilema de especies de ambientes áridos y semiáridos, por lo general, está adaptado para la conducción de agua bajo una alta presión negativa y para evitar los riesgos de bloqueo de la conducción debido a las embolias y cavitación (Lindorf, 1994; Carlquist, 1988). En la conducción del agua influyen el diámetro y la longitud de los elementos de vaso, el tipo de placa de perforación y las punteaduras de las paredes laterales. Los elementos de vaso decrecen en diámetro y longitud, aumentan en frecuencia, en espesor de la pared celular y se agrupan para prevenir el colapso bajo las condiciones de presiones negativas conforme aumenta la aridez (Lindorf, 1994). Esto ha sido considerado como una estrategia para una conducción segura (Baas, 1976; Baas y Carlquist, 1985; Zimmermann, 1982; Zimmermann y Brown, 1971).

El área natural de Aspidosperma quebracho-blanco Schlecht, Apocinácea, una de las especies de mayor importancia comercial en el Parque Chaqueño Seco, se extiende por Argentina, Bolivia, Uruguay y Paraguay. En la Argentina alcanza su mayor extensión y abarca la región del Chaco y parte de la región del Monte y la Mesopotamia (Dimitri et al., 1997). Por su amplia área de distribución y sus características, puede considerársele como una especie sumamente plástica (Tortorelli, 1956). Es una de las dos especies siempreverdes de la Región Chaqueña.

La observación microscópica del xilema de Aspidosperma quebrachoblanco indica que no posee las características anatómicas comunes en especies de zonas áridas y semiáridas, excepto las placas de perforación simple y elementos de vaso cortos. Podría suponerse que la estructura anatómica es desventajosa desde un punto de vista ecológico. En consecuencia debe existir una estrategia adaptativa que le permite contrarrestar esta aparente desventaja de la estructura anatómica.

\section{OBJETIVO}

En este marco, el objetivo de este trabajo es demostrar la interrelación entre los sistemas de conducción y resistencia en su leño como estrategia adaptativa del xilema de la especie que le permite adaptarse a los factores limitantes del ambiente.

\section{METODOLOGÍA}

Se utilizaron 10 ejemplares adultos de Aspidosperma quebracho-blanco Schlecht, con diámetros superiores a los $30 \mathrm{~cm}$, extraídos de dos sitios de la 
Región del Chaco, Santiago del Estero, Argentina. Se escogieron individuos adultos y sanos, sin daños visibles y de fuste recto, según las recomendaciones de Burley y Wood (1979). Sobre el radio norte de la sección transversal se tomaron cinco probetas a $1.30 \mathrm{~m}$ de altura de cada árbol. Las probetas se tomaron a partir de $2 \mathrm{~cm}$ de la médula intercalando un centímetro entre ellas (Bosman et al., 1994). Este diseño de muestreo obedeció a que en este estudio quería estudiarse la interrelación entre un sistema y otro a lo largo de la vida del árbol, abarcando tanto la madera juvenil como madura.

Para la descripción microscópica se utilizó la terminología recomendada por el Comité de la IAWA (IAWA, 1989). Las probetas, orientadas y hervidas 20 horas, se cortaron en espesores de entre 10 y 20 micras, con micrótomo de deslizamiento Leitz. Las secciones transversales, radiales y tangenciales obtenidas, se tiñeron con acridina-crisoidina y azul de astra (Freund, 1970, citado por Vidal y Richter, 1984) deshidratadas en una secuencia creciente de alcoholes y montadas en entellán.

Los macerados se prepararon colocando astillas de madera en una parte de ácido acético glacial y dos partes de peróxido de hidrógeno, según la técnica de Berlyn y Mikshe (1976). Posteriormente fueron teñidos con safranina y montados en entellán.

Se realizaron 25 mediciones para cada uno de los elementos anatómicos. El diámetro de los vasos se determinó con el espesor de la pared incluida. La longitud de fibras y vasos se midió en los macerados. La longitud de miembros de vaso incluye los apéndices.

El Índice de Vulnerabilidad (I.V.) que relaciona diámetro medio de vasos/ frecuencia media de vasos por $\mathrm{mm}^{2}$ se calculó según Carlquist (1988). Este valor indica la seguridad en la conducción del leño. Valores bajos indican seguridad en la conducción y viceversa. El área de vasos se calculó multiplicando el valor del diámetro de los elementos de vasos por la frecuencia media de los mismos por $\mathrm{mm}^{2}$. Este valor se usa como un índice ecológico de la vitalidad del árbol (Sap y Eckestein, 1995); también fue utilizada por Giménez et al. (1994) para caracterizar ecológicamente a Prosopis kuntzei.

Los valores porcentuales de tejidos se obtuvieron siguiendo la metodología de Quirk y Smith (1975). El porcentaje de fibras incluye fibras libriformes, fibrotraqueidas y traqueidas vasicéntricas (Fujiwara et al., 1991).

Las microfotografías se tomaron con equipo LEITZ-OM 100. Los elementos medidos se presentan en la tabla 1.

\section{Análisis estadístico de los datos}

El análisis multivariado permite conocer la estructura de los datos, establecer relaciones y generar hipótesis que expliquen un fenómeno (Afifi y Clark, 1999).

Esta técnica consiste en encontrar varias combinaciones lineales de un conjunto de variables " $Y$ " dependientes y el mismo número de combinaciones lineales del conjunto " $X$ " independientes de tal manera que expresen la mejor correlación de ambos conjuntos:

$$
U_{1}=a_{1} Y_{1}+a_{2} Y_{2}+a_{3} Y_{3}+\ldots+a_{p} Y_{p}
$$

Estas combinaciones lineales son las llamadas variables canónicas y las correlaciones entre los correspondientes pares de variables canónicas se denominan correlaciones canónicas.

$$
V_{1}=X_{1} b_{1}+X_{2} b_{2}+X_{3} b_{3}+\ldots+X_{q} b_{q}
$$


Tabla 1. Elementos anatómicos medidos

\begin{tabular}{ll}
\hline Frecuencia de vasos $\left(\mathrm{n} / \mathrm{mm}^{2}\right)$ & Espesor de pared de fibras $(\mu \mathrm{m})$ \\
Diámetro tangencial de vasos $(\mu \mathrm{m})$ & Diámetro del lumen de las fibras $(\mu \mathrm{m})$ \\
Área de vasos $\left(\mathrm{mm}^{2}\right)$ & Porcentaje de poros $(\%)$ \\
Longitud de elementos de vasos $(\mu \mathrm{m})$ & Porcentaje de fibras $(\%)$ \\
Longitud de fibra $(\mu \mathrm{m})$ & Porcentaje de parénquima $(\%)$ \\
Diámetro tangencial de fibras $(\mu \mathrm{m})$ & Porcentaje de radios $(\%)$ \\
\hline
\end{tabular}

Para estudiar la relación entre los sistemas funcionales del xilema, se utilizó la correlación canónica entre el conjunto de variables que representan el sistema de conducción y el conjunto de variables asociadas con la resistencia mecánica. Esta técnica es especialmente útil en los casos donde existe más de una variable dependiente naturalmente relacionadas, de modo que no tiene sentido tratarlas separadamente (Afifi y Clark, 1999). Para asegurar la adherencia con los presupuestos de los análisis paramétricos y dar mayor confiabilidad a los resultados se realizó el análisis exploratorio de los datos. Como resultado de este análisis, los datos fueron centrados por columnas. Para el análisis de Correlaciones Canónicas se seleccionaron las variables: área de vasos, diámetro de vaso y longitud de elementos de vasos para expresar la función de conducción y, las variables: diámetro de fibras, porcentaje de fibras, espesor de pared y longitud de fibras, asociadas a la resistencia con el supuesto de que ellas también intervienen en la conducción.

Los conjuntos de variables escogidos generaron las siguientes variables canónicas:

$$
\begin{gathered}
U_{1}=\text { A.V. }+ \text { D.V. }+ \text { L.V. } \\
V_{1}=\text { D.F. }+ \text { P.F. E.P. }+ \text { L.F. }
\end{gathered}
$$

Donde:

$D . V .=$ diámetro de vasos

A.V. = área transversal de lúmenes

L.V. = longitud de elementos de vasos

D.F. = diámetro de fibras

L.F. $=$ longitud de fibras

E.P. = espesor de pared

$P . F .=$ porcentaje de fibras

Se obtuvo el Coeficiente de Correlación Canónico que relaciona ambas variables canónicas y los Coeficientes Canónicos Estructurales y Coeficientes de las Variables Canónicas Estandarizados, esto es, la correlación entre cada variable y la variable canónica, para estudiar el sentido y la carga de cada variable en cada conjunto.

\section{RESULTADOS}

\section{Descripción microscópica de la madera de Aspidosperma quebracho-blanco}

Los anillos de crecimiento tienen bordes definidos por 2 a 5 estratos de fibras aplanadas radialmente y con paredes engrosadas tangencialmente (Giménez y Moglia, 1993).

La porosidad es difusa no uniforme (Fig. 1). La frecuencia promedio de vasos es de 9.7 vasos $/ \mathrm{mm}^{2}$ (máx = 18.5 y mín = 4.5). En algunos anillos se observa una gradación en el tamaño y frecuencia de los vasos. 
Los poros son solitarios de forma circular u oval sin contenidos y con placas de perforación simple (Fig. 2). Las punteaduras de los vasos se localizan en los sectores de contacto con otras células debido a que los vasos son solitarios. Las punteaduras de los vasos son alternas lenticulares de tamaño medio y numerosas con valores de abertura externa de $8.5 \mu \mathrm{m}(7-10 \mu \mathrm{m})$ y $4.8 \mu \mathrm{m}$ de abertura interna. El diámetro promedio de los elementos de vasos es de $86.58 \mu \mathrm{m}$ $(40-152 \mu \mathrm{m})$ y se encuentra en la segunda categoría de la clasificación de IAWA. La longitud promedio de los elementos de vasos es $317.48 \mu \mathrm{m}(160-500 \mu \mathrm{m})$; el $50 \%$ de ellos no posee apéndices. La longitud promedio de los apéndices es de $50 \mu \mathrm{m}$ (20-140 $\mu \mathrm{m})$. En algunos ejemplares se observó que la distribución del diámetro de los vasos es bimodal e independiente de la distancia considerada. Los vasos de mayor diámetro tienen $96.58 \mu \mathrm{m}$ en promedio (62.5-152 $\mu \mathrm{m})$, con apéndices muy pequeños o sin ellos, con $25 \mu \mathrm{m}$ (20$40 \mu \mathrm{m}$ ) de longitud (Fig. 3). Los de diámetro pequeño $25 \mu \mathrm{m}(10-45 \mu \mathrm{m})$, son largos y con apéndices muy pronunciados en forma de pico de hasta $140 \mu \mathrm{m}$ de longitud (Fig. 4).

Se observa gran cantidad de traqueidas vasicéntricas con abundantes punteaduras de tamaño y longitud similares a las de los elementos de vaso.

El parénquima es apotraqueal reticulado o difuso en agregados, distribuido en bandas discontinuas de una célula de espesor. Las células de parénquima tienen forma rectangular distribuidas en series de 9 a 15 células de altura.

Los radios son, en su mayoría, tri a tetraseriados con 14 a 15 células de altura. Son homogéneos (Fig. 2). El porcentaje de radios es abundante, constituyendo el $30 \%$ del total de tejidos del xilema. Se observan cristales prismaticos y rómbicos en los radios (Fig. 5).

El tejido fibroso está compuesto por fibras libriformes y fibrotraqueidas. La longitud media de fibras y fibrotraqueidas es de $1407.18 \mu \mathrm{m}$. Son de sección poligonal con un espesor de pared de 6.07 $\mu \mathrm{m}$, paredes gruesas de acuerdo con la clasificación de Fujiwara et al. (1991). El diámetro tangencial medio de fibras es de $16.75 \mu \mathrm{m}$ con un lumen de diámetro medio de $6.1 \mu \mathrm{m}$. Las fibrotraqueidas tienen punteaduras bien desarrolladas de $4.5 \mu \mathrm{m}$ de ancho medio.

En la tabla 2 se resumen los resultados obtenidos.

\section{Correlaciones canónicas}

Los resultados de este análisis revelan que el conjunto de variables seleccionadas para representar a la conducción está altamente correlacionada con aquel que representa la resistencia mecánica en el xilema $\left(R^{2}=0.694\right)$ con un $\mathrm{Ji}$ cuadrado altamente significativo (Tabla 3). También expresa que del $100 \%$ de la variabilidad extraída de las variables dependientes, el $79.45 \%$ lo explican las variables independientes.

Los Coeficientes Canónicos Estructurales y los Coeficientes Canónicos Estandarizados no son iguales en valor debido a que las variables están interrelacionadas (Afifi y Clark, 1999). De acuerdo con el autor, cuando los conjuntos de las variables son independientes entre sí, los coeficientes canónicos y los estructurales son iguales. Se utilizaron los coeficientes estructurales de las variables canónicas que permiten una interpretación más simple del fenómeno biológico (Afifi y Clark, 1999). El sentido y el peso de las variables originales sobre las variables canónicas se expresan en la tabla 4.

Los coeficientes canónicos estructurales determinan las siguientes variables canónicas:

$$
\begin{gathered}
U_{1}=+0.66 \text { A.V. }+0.59 D . V .-0.45 L . V . \\
V_{1}=0.97 D . F .+0.57 \text { P.F. }-1.19 E . P .-0.157 L . F .
\end{gathered}
$$




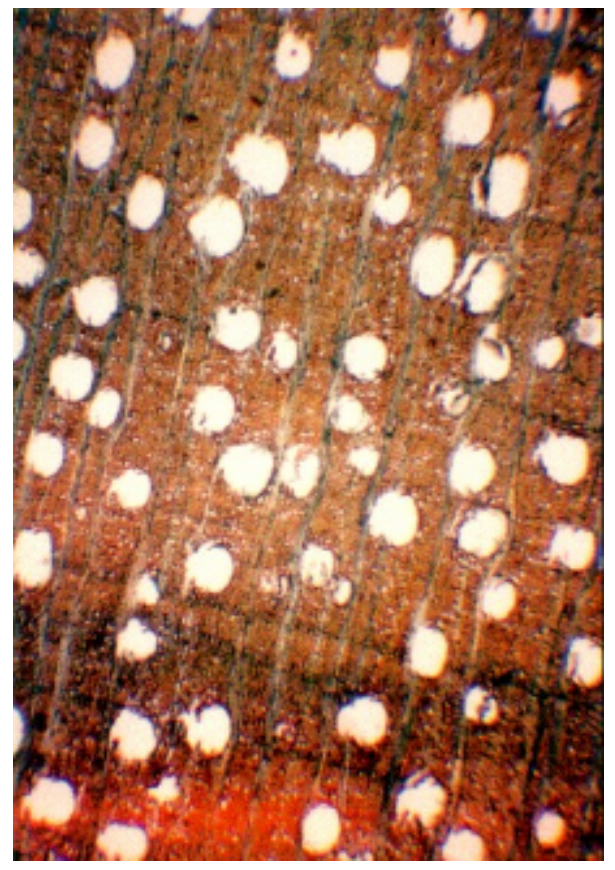

Figura 1. Corte transversal X100 A Poros solitarios

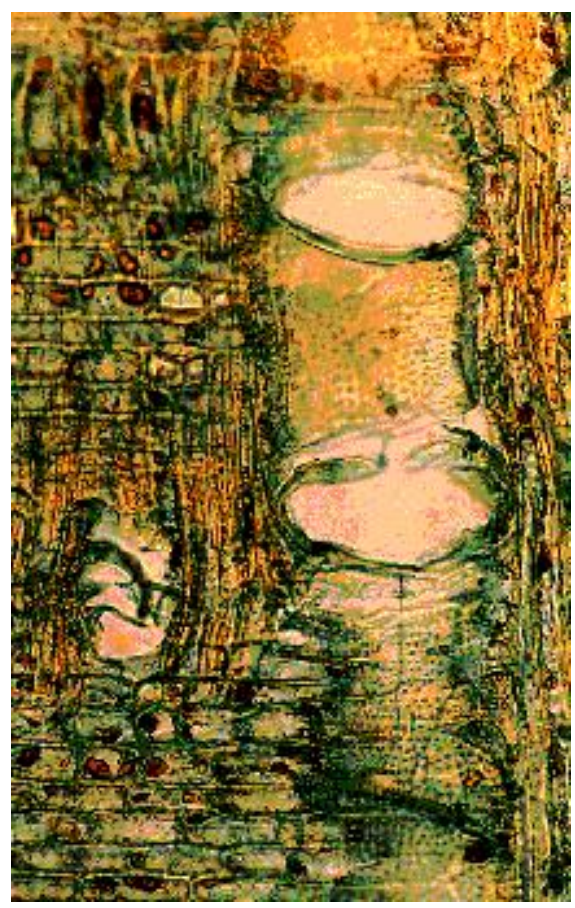

Figura 2. Corte radial, radios homogéneos X250 A

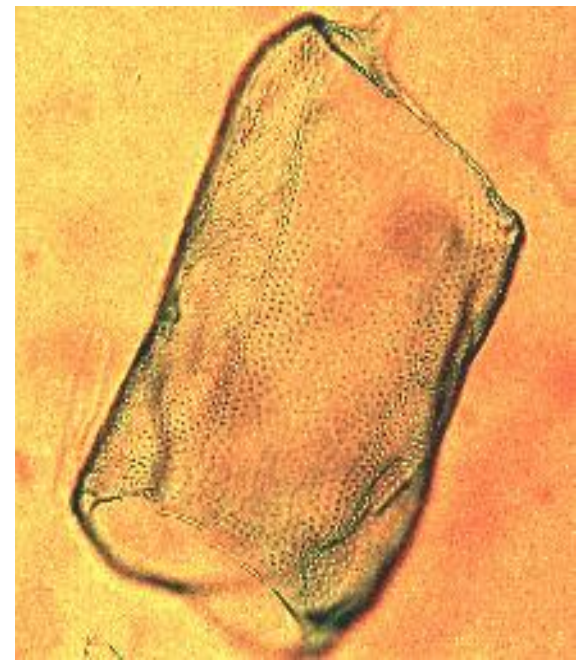

Figura 3. Miembro de vaso corto X250 A

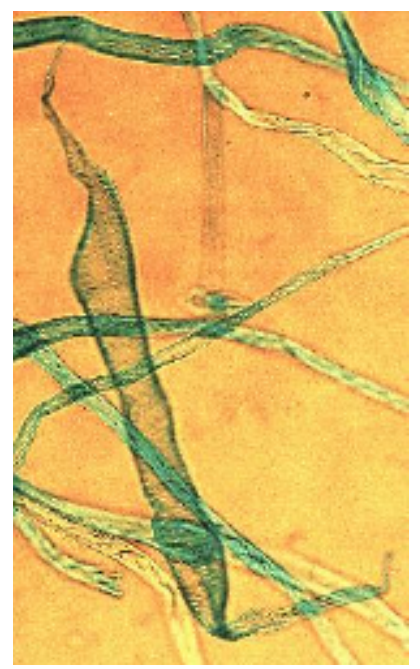

Figura 4. Elemento de Vaso muy largo similar a traqueidas

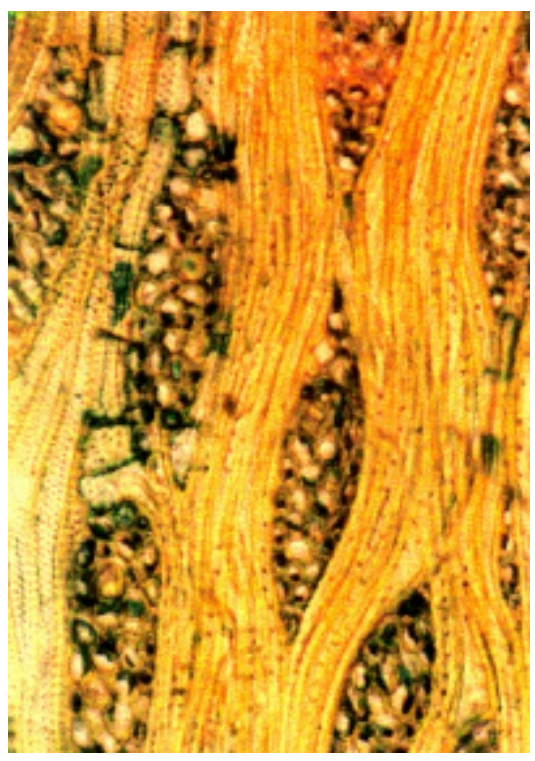

Figura 5. Sección tangencial X250 A con abundantes fibrotraqueidas, cristales en las células de los radios 
Tabla 2. Valores promedio y coeficiente de variación de las variables medidas

\begin{tabular}{lccc}
\hline VARIABLES & $\begin{array}{c}\text { PROMEDIO } \\
\text { GENERAL }\end{array}$ & $\begin{array}{c}\text { DESVIACIÓN } \\
\text { ESTÁNDAR }\end{array}$ & $\begin{array}{c}\text { COEFICIENTE } \\
\text { DE VARIACIÓN } \\
(\%)\end{array}$ \\
\hline Frecuencia de vasos $\left(\mathrm{n} / \mathrm{mm}^{2}\right)$ & 9.77 & 1.54 & 15.79 \\
\hline Diámetro tangencial de vasos & $86.58 \mu \mathrm{m}$ & 12.09 & 13.97 \\
\hline Área de vasos & $0.2 \mathrm{~mm}^{2}$ & 0.06 & 31.77 \\
\hline Longitud de elementos de vaso & $317.48 \mu \mathrm{m}$ & 30.99 & 9.76 \\
\hline Longitud de fibra & $1411 \mu \mathrm{m}$ & 245.5 & 17.39 \\
\hline Diámetro tangencial de fibras & $16.58 \mu \mathrm{m}$ & 0.69 & 4.14 \\
\hline Espesor de pared de fibras & $6.07 \mu \mathrm{m}$ & 0.46 & 7.62 \\
\hline Diámetro del lumen de las fibras & $5.37 \mu \mathrm{m}$ & 0.37 & 6.99 \\
\hline Porcentaje de poros & 15.03 & 3.18 & 21.14 \\
\hline Porcentaje de fibras & 48.18 & 8.33 & 17.29 \\
\hline Porcentaje de parénquima & 9.1 & 2.99 & 32.89 \\
\hline Porcentaje de radios & 27.6 & 7.23 & 26.23 \\
\hline
\end{tabular}

Tabla 3. Resumen de la correlación canónica

\begin{tabular}{|c|c|c|}
\hline \multicolumn{3}{|c|}{ Canonical $\mathbf{R}^{2}: 0.69498$} \\
\hline \multirow[t]{2}{*}{$\mathrm{Chi}^{2}(12)=36.604$} & \multicolumn{2}{|l|}{$p=0.00026$} \\
\hline & $\begin{array}{c}\text { VARIABLE } \\
\text { CANÓNICA U }\end{array}$ & $\begin{array}{c}\text { VARIABLE } \\
\text { CANÓNICA V }\end{array}$ \\
\hline Varianza extraída & $100.000 \%$ & $79.4599 \%$ \\
\hline \multirow[t]{5}{*}{ Total redundancia } & $25.9276 \%$ & $18.9492 \%$ \\
\hline & D. V. & L. F. \\
\hline & A.V. & D.F. \\
\hline & L. V. & E.P. \\
\hline & & P.F \\
\hline
\end{tabular}


Tabla 4. Coeficientes canónicos estructurales

\begin{tabular}{|c|c|c|c|}
\hline \multicolumn{4}{|c|}{ COEFICIENTES CANÓNICOS DE LA VARIABLE U } \\
\hline & Correlación1 & Correlación 2 & Correlación 3 \\
\hline D.V. & 0.5956 & 0.5889 & -0.9662 \\
\hline A. $V$. & 0.6600 & -0.5014 & 0.8354 \\
\hline L. V. & -0.4577 & 0.7176 & 0.7407 \\
\hline \multicolumn{4}{|c|}{ COEFICIENTES CANÓNICOS DE LA VARIABLE V ${ }_{1}$} \\
\hline & Correlación 1 & Correlación 2 & Correlación 3 \\
\hline F. L. & -0.1571 & 0.8636 & -0.1712 \\
\hline F. D. & 0.9703 & 0.3433 & 0.2953 \\
\hline E. P. & -1.1904 & -0.1352 & -0.4601 \\
\hline P. F. & 0.5695 & -0.3840 & -0.7938 \\
\hline
\end{tabular}

\section{DISCUSIÓN}

El quebracho blanco tiene porosidad difusa. Moglia y Giménez (1998), determinaron que el $43 \%$ de 24 especies estudiadas del Chaco, poseen porosidad difusa no uniforme.

Bissing (1982) determinó que la porosidad es el carácter que más varía en función de la disponibilidad de agua. Las especies de hábitats xéricos modifican su porosidad difusa uniforme a porosidad difusa no uniforme al ser cultivadas en ambientes más húmedos. Aunque el quebracho blanco tiene una marcada diferencia en la frecuencia y distribución de los vasos, no pudo establecerse que esta variabilidad se debiera a una diferencia entre sitios con distintos balances hídricos (Moglia, 2000).

La porosidad difusa tiene implicaciones fisiológicas. El patrón de transporte de agua es muy diferente si una especie tiene porosidad anular o difusa (Zimmermann, 1983). En el primer caso el transporte de agua se realiza a través de los vasos grandes del leño temprano del último anillo. En las especies de porosidad difusa la conducción es a través de varios anillos. También hay diferencias en los patrones de diferenciación del xilema (Suzuki et al., 1996). Los primeros vasos en este tipo de especies, se diferencian más tardíamente, luego del brote de las yemas apicales.

Existen ciertos caracteres estructurales del xilema que aparecen con mayor frecuencia en las plantas de zonas áridas y semiáridas y que son señalados por diversos autores (Baas y Carlquist, 1985; Carlquist y Hoeckman, 1985; Barajas, 1985) como ventajosos ya que confieren mayor seguridad en la conducción del agua. Estos caracteres son: alta frecuencia de poros por unidad de superficie, poros agrupados, tamaño reducido de sus elementos, vasos con placas de perforación simple y engrosamientos en las paredes de los vasos. La observación microscópica del xilema de Aspidosperma quebracho blanco revela que no posee estas características, excepto las placas de perforación simple (que permite una 
óptima conducción) y los elementos de vaso cortos.

El Índice de Vulnerabilidad (I.V.) caracteriza la seguridad o vulnerabilidad del leño con base en los elementos de vasos. El I.V. es 8.7 para el quebracho blanco, lo cual es alto si se compara con las especies xeromorfas del Chaco. Éstas además tienen vasos solitarios, como Maytenus vitis idaea (I.V. 0.31), Zizyphus mistol (I.V. 3.40) (Moglia y Jiménez, 1998) - Aspidosperma cuspa (I.V. de 0.11) (Lindorf, 1994), especie del mismo género de una región semiárida con características más xeromorfas en su leño. Las tres especies mencionadas tienen alta frecuencia de vasos. Puede suponerse que la estructura anatómica es desventajosa desde un punto ecológico. Es evidente que el tejido vascular no proporciona seguridad en esta madera, sino que debe existir un sistema de conducción subsidiario.

La presencia casi exclusiva de vasos solitarios con tejido de resistencia compuesto principalmente por fibrotraqueidas y traqueidas vasicéntricas está citada como una característica primitiva por Wheeler y Baas (1991), aunque Carlquist (1988) sostiene que la presencia de traqueidas vasicéntricas ofrece un tejido conductivo subsidiario que hace superflua la presencia de vasos agrupados. En quebracho blanco, se ha observado que algunos de los elementos finales de vasos (Fig. 6) terminan en contacto con las traqueidas (Fig. 7). Zimmermann (1982) observó que los vasos finalizan en la mayor parte de los casos en pares o en grupos. Esto tiene sentido funcional ya que el agua se mueve de un vaso a otro, vía punteaduras intervasculares.

La presencia exclusiva de vasos solitarios asociada a traqueidas vasicéntricas y fibrotraqueidas como el tejido de resistencia, se ha registrado en las floras de diferentes regiones semiáridas (Carlquist, 1988; Barajas, 1985; Lindorf,

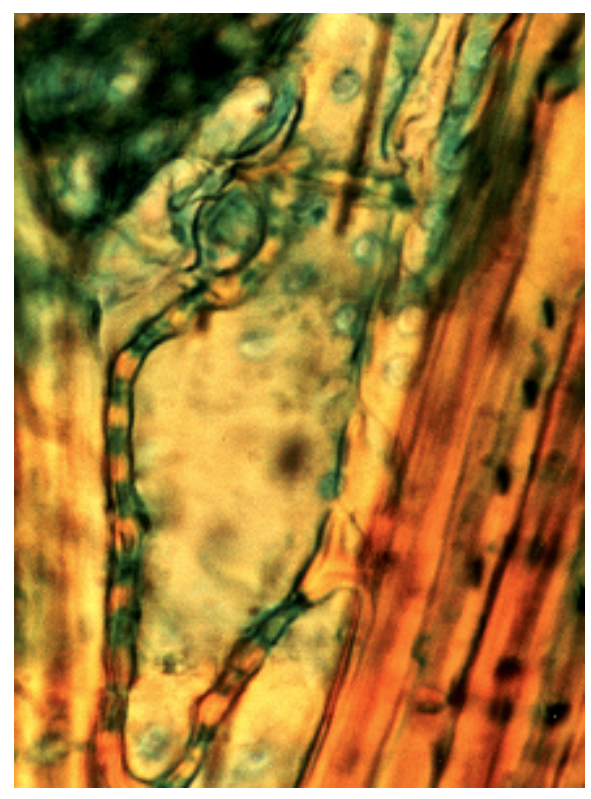

Figura 6. Sección tangencial $\times 1000$ elemento final de un vaso con pared final en contacto con traqueida

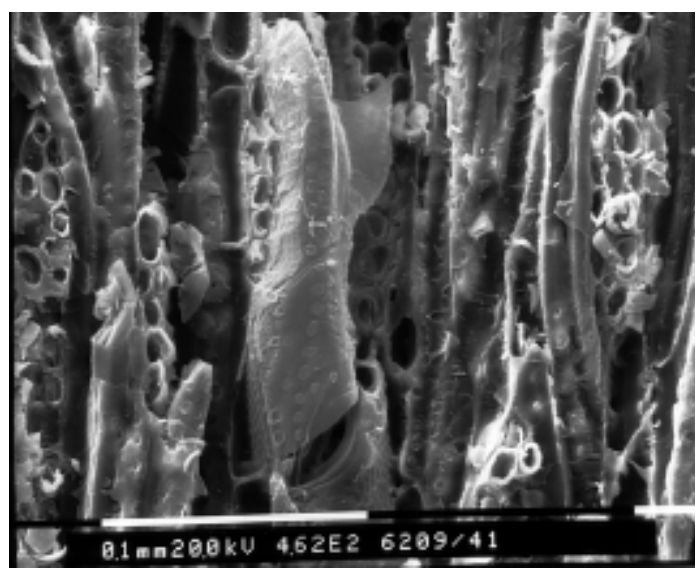

Figura 7. Sección tangencial X462 E2. Elemento final de un vaso

1994; Moglia y Giménez 1998). Carlquist (1988) confirma la correlación en su compilación de datos sobre Dicotiledóneas, estableciendo que en todas las familias con vasos solitarios hay traqueidas, como ejemplo Mirtáceas, Proteaceas o en el género Quercus. En la Región Chaqueña, Moglia y Giménez (1998), encontraron una relación inversa entre el aumento de presencia de traqueidas vasicéntricas y la disminución de poros agrupados. 
Las traqueidas son células adaptadas a un transporte seguro durante un déficit hídrico. Según Ellmore y Ewers (1985) los vasos pequeños se embolizan más lentamente que los grandes y las traqueidas aún más, por lo que son más seguras. Además, no sólo es importante la presencia de las traqueidas sino su distribución. Al ser vasicéntricas, es decir, adyacentes a los elementos de vasos y distribuidas uniformemente, forman una red tridimensional de columnas de agua en todo el tallo que permite proveer de agua a toda la copa. Este tipo de traqueidas caracteriza, según Carlquist (1988), a especies de follaje perenne y que habitan en áreas con marcado estrés de agua como es el caso de quebrachoblanco.

La presencia de fibrotraqueidas para la familia Apocinácea está citado por Carlquist (1985). Aunque algunos autores como Bailey (1936) y Carlquist (1988), consideran que las fibrotraqueidas no son esencialmente conductivas (ya que son elementos intermedios entre las fibras y traqueidas), la naturaleza no conductiva de las fibrotraqueidas todavía es materia de discusión y se basa en la semejanza de estos elementos con las fibras, más que con las traqueidas y sin tomar en cuenta las punteaduras que, en general, son semiareoladas.

Varios autores sostienen que las fibras con punteaduras areoladas pueden servir para proveer de un sistema subsidiario conductivo, con seguridad adicional (Braun, 1961; Carlquist, 1988; Baas y Schweingruber, 1987). Wheeler y Baas (1991) encontraron que un gran porcentaje de la flora fósil hallada hasta el presente tenía este tipo de fibras.

Las fibrotraqueidas observadas en el quebracho-blanco tienen punteaduras ornamentadas muy abundantes por lo que es muy probable que también puedan conducir o por lo menos tengan una intervención menor en la seguridad en la conducción. El xilema de esta especie es una intrincada red tridimensional inter- conectada. La conducción radial está asegurada por la gran cantidad de radios, con todas sus células de contacto.

Los resultados del análisis de correlaciones canónicas permitieron confirmar que tanto los elementos de conducción como los de resistencia se encuentran íntimamente relacionados.

La relación entre ambos grupos de variables, sugiere que es mejor interpretar el hidrosistema en conjunto, pues su estrategia adaptativa no sería la diferenciación de funciones sino la interrelación de los elementos. El hidrosistema del quebracho blanco está constituido por vasos solitarios que transportan los volúmenes importantes de agua y un sistema subsidiario altamente eficiente en realizar una conducción segura a través de las traqueidas con una seguridad adicional dada por las fibrotraqueidas con punteaduras areoladas. La estructura anatómica del quebracho blanco concuerda con la interpretación de Carlquist (1988) sobre el funcionamiento de las traqueidas, en el sentido de que los vasos pueden conectarse a través de ellas en agregados diagonales, los cuales incorporan a la mayoría de los vasos, convirtiéndose en grupos intercontínuos, transformando el xilema en una red intercomunicada, (Carlquist, 1988).

Este sistema interconectado es una buena adaptación al medio que le ha permitido al quebracho blanco tener una amplia zona de distribución y extenderse más allá de los límites de la región Chaqueña, donde las condiciones ambientales son más severas, como en la región del Monte.

\section{CONCLUSIONES}

Los resultados obtenidos justifican las siguientes conclusiones:

- Los conjuntos de variables seleccionados presentan una alta correlación 
demostrando que los sistemas de conducción y resistencia se encuentran interrelacionados. Las traqueidas vasicéntricas y fibrotraqueidas intervienen en la conducción y forman un sistema subsidiario que coadyuva al sistema principal. En esta especie, el xilema funciona a manera de una red interconectada.

- El quebracho blanco presenta un tejido xilemático altamente especializado en tolerar el estrés hídrico, desarrollando una estrategia adaptativa diferente a la mayoría de las especies de la Región Chaqueña.

- Ulteriores estudios sobre el patrón de foliación, la anatomía foliar y su sistema radicular ayudarán a una interpretación fisiológica integral del hidrosistema del árbol.

\section{REFERENCIAS}

Afifi, A.A. y V. Clark. 1999. ComputerAided multivariate analysis. Third Ed. Texts in Statistical Science. Chapman y Hall CRC. Boca Raton, Londres, Nueva York, Washington D.C. $455 \mathrm{p}$.

Baas, P. y S. Carlquist. 1985. A comparison of ecological wood anatomy of the floras of southern California and Israel. IAWA 8: 245274.

Baas, P. y F. Schweingruber. 1987. Ecological trends of trees, shrubs, and clim-bers from Europe. IAWA 8: 245274.

Bailey, I.W. 1936. The problem of differentiation and classification of tracheids, fiber-tracheids, and libriform fibers. Trop. Woods 45: 1823.

Barajas M., J. 1985. Wood structural differences between trees of two tropical forests in Mexico. IAWA 8: 355-364.

Berlyn, B. y J. Mishke. 1976. Botanical Micro thecnique and Cythochemestry. lowa State University. Ames lowa.

Bissing, D.R. 1982. Variation in qualitative anatomical features of xylem of selected dicotyledonous woods in relation to water availability. Bulletin of the Torrey Botanical Club 109: 371-384.

Bosman, M., I. Kort,M. van Genderen, y P. Baas. 1994. Radial variation in wood properties of natural and plantation grown light red meranti (Shorea, Dipterocarpaceae). IAWA 15: 111120.

Braun, H.J. 1961. The organization of the Hydrosystem in the stemwood of trees and shrubs. IAWA 1:2-9.

Burley, J. y P.J. Wood. 1979 Manual sobre investigaciones de especies y procedencias con referencia especial a los trópicos. Tropical Forestry Papers 10. Décima ed. Departament of Forestry. Commonwealth Forestry Institute. University of Oxford.

Carlquist, S. 1985. Vasicentric tracheids as drought survival mechanism in the woody flora of southern California and similar regions; review of vasicentric tracheids. Aliso 11: 37-68.

Carlquist, S. 1988. Comparative wood anatomy. Springer Series of wood Science. Springer Verlag. Nueva York. $436 \mathrm{p}$.

Carlquist, S. y D.A. Hoekman. 1985. Ecological wood anatomy of the woody Southern California flora. IAWA 6: 319-347.

Dimitri, M.J., F. Rosario, J. Leonardis y J.S. Biloni. 1997. El nuevo libro del 
árbol. Tomo I. Editorial El Ateneo. $190 \mathrm{p}$.

Ellmore, G.S. y F.W. Ewers. 1985. Hydraulic conductivity in trunk xylem of elm, Ulmus americana. IAWA 6: 303-307.

Freund, H. 1970. Handbuch der Mikroscopie in der Techknik. Frankfurt: Umschan Verlag. 379 p.

Fujiwara, S., K. Sameshima, K. Kuroda y N. Takamura. 1991. Anatomy and properties of japanese hardwoods I. Variation of fiber dimensions and tissue proportions and their relation to basic density. IAWA 12(4): 419424.

Giménez, A.G. y J.G. Moglia. 1993. Determinación de crecimiento de especies leñosas arbóreas de la Región Chaqueña Seca. Revista de Difusión Científica y Tecnológica de la Facultad de Ciencias Forestales, Universidad Nacional de Misiones. Yvyrareta 4(4):46-60.

Giménez, A., G. Moglia y M. Juárez. 1994. Variabilidad ecológica de Prosopis kuntzei: Influencia del déficit hídrico en la estructura interna. Actas del Congreso Forestal Argentino y Latinoamericano. Comisión V. Bosques Nativos (1):67-75.

IAWA Committee 1989. IAWA List of microscopic features for hardwood identification. IAWA Bulletin n.s. 10 (3): 219-332. In: Wood Structure in Biological and technological research (eds. P. Baas A.J. Bolton y D.M. Catling) 157-181. Leiden Botanical Series No. 3. Leiden Univ. Press.

Lindorf, H. 1994. Eco-anatomical wood features of species from a very dry tropical forest. IAWA 15(4):361-376.

Moglia, J.G. y A.M. Jiménez. 1998. Rasgos anatómicos característicos del hidrosistema de las principales especies arbóreas de la Región Chaqueña Argentina. Revista de Investigación Agraria Sistema y Recursos Forestales 7 (1) y (2): 53-71.

Moglia J.G. 2000. Variabilidad radial de los caracteres anatómicos de Aspidosperma quebracho-blanco Schelkt, Apocinaceae. Tesis para la obtención del grado de Doctor. Universidad Nacional de Tucumán. 95 p.

Quirk, J. y D. Smith. 1975. Comparison of dual linear and dot grid eyepiece methods for estimating wood properties of Douglas - fir. Wood Sci. 8: 92-96.

Sab, U. y D. Eckestein. 1995. The variability of vessel size in beech (Fagus sylvatica L.) and its ecophysiological interpretation. Trees 9: 123-145.

Suzuki, M.,Y. Kiyotsugu y H. Suzuki 1996. Phenological comparison of the onset of vessel formation between ringporous and diffuse-porous deciduous trees in a Japanese temperate forest. IAWA 17 (4): 431-444.

Vidal G., A. y H.G. Richter. 1984. Microtécnica e fotomicrografia. Curso de Pós-Graduaçao em Engenharia Florestal. Universidade Federal do Paraná. Curitiba-Brasil.

Tyree, M.T.,S.D. Davis y H. Cochard 1994. Biophysical perspectives of xylem evolution: is there a tradeoff of hydraulic efficiency for vulnerability to dysfunction? IAWA 15: 335-360.

Tortorelli, L.A. 1956. Madera y Bosques Argentinos. Bs. As. 910 p.

Wheeler, E. y P. Baas. 1991. A survey of the fossil record for dicotyledonous wood and its significance for evolutionary and ecological wood anatomy. IAWA 12: 271-332. 
Zimmermann, M.H 1982. Functional xylem anatomy of angiosperm trees. $P$. Baas (ed). New perspectives in wood anatomy: 59-70. Nijhoff/Junk.

\section{Zimmermann, M.H. 1983. Xylem structure and the ascent of sap. Springer Verlag, Berlin. $125 \mathrm{p}$.
Zimmermann, M. H. y C. Brown. 1971. Trees Structure and Function. Springer, Berlin. 336 p. $\diamond$

1 Instituto de Silvicultura y Manejo de Bosques Facultad de Ciencias Forestales. Universidad Nacional de Santiago del Estero. Av. Belgrano 1912 (S), 4200 Santiago del Estero. Argentina. c.e.: vimog@unse.edu.ar

Manuscrito recibido el 1 de agosto de 2000

Aceptado el 11 de diciembre de 2000

Este documento se debe citar como:

Moglia, J.G. y C.R. López. 2001. Estrategia adaptativa del leño Aspidosperma quebracho-blanco. Madera y Bosques 7(2):13-25. 